\title{
$\widehat{A}$ Madridge \\ madridge Journal of Vaccines \\ interconnecting Scientific world
}

Editorial Article

Open Access

\section{A Novel potential Adjuvant for Cancer Vaccines}

\author{
Marco Ruggiero $^{\star}$ and Stefania Pacini \\ Silver Spring Sagl, 6864 Arzo, Switzerland
}

\section{Article Info}

*Corresponding author:

Marco Ruggiero

Silver Spring Sagl,Via R. Rossi 24

6864 Arzo, Switzerland

E-mail: marco.drruggiero@gmail.com

Received: September 18, 2018

Accepted: September 24, 2018

Published: September 29, 2018

Citation: Ruggiero M, Pacini S. A Novel potential Adjuvant for Cancer Vaccines. Madridge J Vaccines. 2018; 2(1): 57-61. doi: $10.18689 / \mathrm{mjv}-1000112$

Copyright: ( $\odot 2018$ The Author(s). This work is licensed under a Creative Commons Attribution 4.0 International License, which permits unrestricted use, distribution, and reproduction in any medium, provided the original work is properly cited.

Published by Madridge Publishers

\begin{abstract}
In this Editorial, I describe a novel potential adjuvant for cancer vaccines designated imuno ${ }^{\mathrm{TM}}$, an emulsion of low molecular weight microbial chondroitin sulfate, phosphatidylcholine and vitamin $D_{3}$. The molecules constituting imuno ${ }^{T M}$ are arranged in cell membrane-like structures under the form of a homogeneous, single-phospholipid bilayer that resembles protocellular structures with chondroitin sulfate mimicking the role of nucleic acids. Phosphatidylcholine and chondroitin sulfate, arranged in protocellular-like structures, have to be interpreted as a universal delivery system with the potential of maximizing the effects of vaccination as we proposed for HIV DNA vaccines; such a molecular arrangement proves essential for the functioning of imuno ${ }^{\mathrm{TM}}$ as a novel type of cancer vaccine adjuvant.
\end{abstract}

Keywords: Cancer; Vaccines; Immunotherapy; Adjuvant; GcMAF; Chondroitin sulfate.

\section{Cancer vaccines}

Until recently, vaccines were considered almost exclusively for prevention and cure of infectious diseases based on the century-old observation that attenuated microbes and/or their antigens, stimulate the immune system so to develop adaptive immunity against a specific pathogen. This concept is based on the assumption that a pathogen is recognized by immune cells as "non-self" and, therefore, as a target to be eliminated through one or more of the arms of the immune system. In the context of oncology, the task of the immune system is definitely more difficult since cancer cells have the ability to "conceal" their identity and escape immune surveillance through a variety of strategies. When cancer arises as a pathologic entity it is precisely because cancer cells have been successful in avoiding immune surveillance.

The idea of vaccination against cancer is an old one that, in modern times, dates back to the nineteenth century and Dr. William B. Coley with his toxin, often referred to as Coley's vaccine. It is worth mentioning, however, that in the times of Coley, knowledge of oncology and immunology were rudimentary at best, and Coley's vaccine was interpreted more as a form of bacterial therapy rather than a form of what today is called cancer immunotherapy. Coley had not been the first to observe an association between bacterial infection and cancer regression; even without counting reports from ancient Egypt and Pharaoh Imhotep in 2,600 BC, in the thirteenth century AD, Peregrine Laziosi, later canonized as the Saint Patron of cancer patients, described spontaneous regression of a large tumor in his leg after the tumor became severely infected and, since the eighteenth century, intentional infection of cancers was considered standard of care treatment that yielded significant successes up to complete regression of advanced cancers [1,2]. With today's knowledge of the functioning of the immune system, we may interpret those results as a brutal stimulation of the immune system that, associated with the unavoidable hyperthermia due to the severity of the infection, led to cancer cell apoptosis and, eventually, to cancer regression.

Cancer vaccines of the twenty-first century are obviously more targeted and less risky than those heroic attempts of the pre-antibiotic era as they try to "educate" the immune system to recognize cancer cells as non-self so to restore immune surveillance 
and efficiently eliminate abnormally proliferating cells. Modern vaccines against cancer have an unquestionable appeal over treatments such as surgery, radiation therapy or chemotherapy, since they are perceived as much less invasive and, at least in theory, they could protect against a specific type of cancer for life just like a vaccination against microbial infections does. Based on these premises, many potential vaccines against specific cancers have been developed, and the phase II/III clinical trials are underway to test their clinical efficacy [3]. At variance with the heroic attempts of the past, today's cancer vaccines are based on antigens derived from self-molecules rather than on infectious pathogens; however, just like in the past, they have been used primarily in advanced, metastatic, cancer with the target of stimulating the immune system so as to slow down the progression of the disease. In other words, until recently, cancer vaccines and, more broadly, cancer immunotherapy, was relegated to those cases where standard of care had failed or was not applicable; it is only in recent years that cancer vaccines and immunotherapy are being interpreted as means to target early cancer and premalignant lesions with the objective of preventing the onset of malignancies [4]. Therefore, two types of cancer vaccines are being developed: therapeutic vaccines that are used in patients who already have cancer, and preventive vaccines aimed at preventing cancer from occurring.

An interesting cancer vaccine that has been licensed for use in clinical practice is Sipuleucel-T (commercial designation, Provenge), a vaccine intended for the treatment of asymptomatic/minimally symptomatic metastatic castrationresistant prostate cancer. This vaccine ultimately relies upon activation of macrophages; to this end, peripheral blood mononuclear cells that are taken from the patient and incubated with a fusion protein consisting of recombinant prostate acid phosphatase and granulocyte-macrophage colony-stimulating factor (GM-CSF) [5]. It is worth noticing that we were the first to demonstrate that a low molecular weight acid phosphatase has phosphotyrosine phosphatase activity, thus counteracting aberrant signaling in cancer cells and inhibiting cancer cell proliferation $[6,7]$. Likewise, we were among the first to highlight the role of macrophage colony stimulating factors in human cancer [8], and to propose macrophage-based immunotherapy in advanced cancer [9]. It is based on this decade-old, proven expertise in the field of cancer cell signaling and therapy that we developed imuno ${ }^{\mathrm{TM}}$, a novel tool that has the potential to boost the efficacy of existing cancer vaccines as well as to stimulate the innate immune system in the context of cancer immunotherapy.

\section{Adjuvants for cancer vaccines}

Cancer vaccines, just like other vaccines, benefit from the addition of adjuvants, compounds that optimize the immune response by increasing the production of antibodies and offering a longer-lasting coverage, thus reducing the amount of antigen that needs to be injected. In addition to increasing antibody production, adjuvants are used to potentiate cellmediated immune responses, for example, by activating T-lymphocytes. Among different types of compounds,
Freund's adjuvant merits special consideration. It was first described in 1942 when Jules Freund and Katherine McDermott published a study describing the immunological response following injection of a "lanolin-like substance and killed tubercle bacilli suspended in oil" in guinea pigs [10]. There exist two types of Freund's adjuvant, the complete and the incomplete form. The former consists of inactivated and dried microbes (typically Mycobacterium tuberculosis) in an emulsion of water and mineral oil whereas the incomplete form lacks the microbial component. The mechanism of action of Freund's adjuvant involves stimulation of cellmediated immunity as well as activation of innate immune responses, thus boosting the efficacy of vaccination. It is worth noticing, however, that use of the original formulation of Freund's adjuvant in humans is forbidden because of toxicity, mainly due to the presence of mineral oil.

In the context of cancer, adjuvants assume particular relevance because most cancer vaccines are poorly immunogenic per se and cancer patients frequently have deficient immune responses, thus making the presence of an adjuvant a mandatory requirement [11]. Although the original formulation of Freund is unsuitable for human use, nevertheless the main tenet of Freund's adjuvant, that is the presence of an emulsion of water and oil, is maintained; for example, Authors from Cambridge (UK) reported in 2012 that a combination of adjuvants based on emulsions of lipophilic compounds elicited immunological responses comparable to Freund's adjuvant [12]. Even though many adjuvants are based on emulsions of water and oil, or water and lipophilic compounds, also other combinations of molecules are efficient cancer vaccine adjuvants. In 2013 Authors from China and the USA demonstrated that polysaccharides may prove useful as cancer vaccine adjuvants with particular reference to advanced, metastatic cancer [13]. Therefore, based on our previous expertise in the field of polysaccharide research in experimental oncology, when we designed imuno ${ }^{\mathrm{TM}}$ we combined the two most effective approaches in the field of adjuvants developing an emulsion of water and a lipophilic molecule complexed with a polysaccharide endowed with immune stimulating properties.

\section{Design of a novel tool for adjuvant immunotherapy}

The design of imuno ${ }^{\mathrm{TM}}$ takes inspiration from our longstanding expertise in experimental oncology as well as from the pioneering work of Dr. Prudden who demonstrated, since 1985, that a polysaccharide, the glycosaminoglycan chondroitin sulfate, is the powerful immune stimulating agent responsible for the anticancer effects of cartilage formulations [14]. Chondroitin sulfate is composed of alternate units of glucuronic acid and $\mathrm{N}$-acetyl-galactosamine, the latter being the active site of the Gc protein-derived Macrophage Activating Factor (GcMAF) [15], a macrophage activating factor endowed with significant adjuvant activity [16]. In 2013, based on molecular modeling, we hypothesized that the immune stimulating properties of GCMAF were due to its association with lipophilic compounds, namely oleic acid, and vitamin $D_{3^{\prime}}$ thus reinforcing the hypothesis that the physical 
features of an emulsion were involved in the biological and clinical effects described for GcMAF [17]. In 2016, in the attempt to solve a number of controversies surrounding GcMAF and its role in immunotherapy, we hypothesized that chondroitin sulfate was indeed responsible for the biological and clinical effects attributed to GCMAF [18]. Based on these observations, we designed an emulsion of chondroitin sulfate, oleic acid and vitamin $D_{3}$ designated Rerum ${ }^{\circledast}$, that proved useful in a variety of conditions where potentiation of the immune system is sought after [19].

Although both GcMAF associated with oleic acid, and Rerum ${ }^{\circledR}$ have shown remarkable effects in immunotherapy [920], the extractive nature of their main components has prevented their widespread use. Thus, GcMAF is synthesized from a blood protein, whereas, until recently, chondroitin sulfate was extracted from animal cartilage and it is well known that compounds extracted from complex matrices suffer from less than optimal homogeneity, purity, and consistency. Because of this, in the design of imuno ${ }^{\mathrm{TM}}$ we took advantage of the recent development of a novel form of chondroitin sulfate that derives from microbial fermentation and shows a degree of purity and homogeneity far superior to that of the animal-derived counterpart [21]. Microbial chondroitin sulfate shows a homogeneous sulfation profile that is much closer to that of human chondroitin sulfate and features a homogeneous low molecular weight whereas animal-derived chondroitin sulfate is a heterogeneous mixture of species with different molecular weights. This feature is of utmost importance as it has been widely demonstrated that low molecular weight glycosaminoglycans are much more effective than their high molecular weight counterparts as it is the case, for example, of heparin [22]. Consistent with this concept, Volpi et al. recently reported that low molecular weight microbial chondroitin sulfate shows far superior bio-availability and pharmacokinetics with a significantly greater clinical efficacy as compared with the animal-derived counterpart. Therefore, imuno ${ }^{\mathrm{TM}}$ is constituted by an emulsion of low molecular weight microbial chondroitin sulfate complexed with phosphatidylcholine and vitamin $D_{3}$. At variance with Rerum $^{\circledR}$, phosphatidylcholine rather than oleic acid was chosen to provide the lipophilic moiety of the supramolecular assembly. The rationale for this choice lays in our decade-old observation that circulating glycosaminoglycans are associated with phosphatidylcholine and this association may be responsible for a number of biological effects [23]. In this way, the molecules constituting imuno $^{\mathrm{TM}}$ are arranged in cell membrane-like structures under the form of a homogeneous, single-phospholipid bilayer that resembles protocellular structures with chondroitin sulfate mimicking the role of nucleic acids [24]. In a broader context, phosphatidylcholine and chondroitin sulfate, arranged in protocellular-like structures, have to be interpreted as a universal delivery system with the potential of maximizing the effects of vaccination as we proposed for HIV DNA vaccines [25]; such a molecular arrangement proves essential for the functioning of imuno ${ }^{\mathrm{TM}}$ as a novel type of cancer vaccine adjuvant.
As a matter of fact, despite the use of adjuvants in billions of doses of human and animal vaccines with undisputable efficacy, their mechanism of action at the molecular level remains the "immunologist's little dirty secret" [26]; many different mechanism have been proposed to solve the "secret" consisting in the fact that exposure to a foreign antigen per se is insufficient to mount an efficient immune response and rather non-specific substances such as mineral oil, killed mycobacteria, or even metals - aluminum - have to be added in order to activate T- and B-lymphocytes. The question is central to understanding the functioning of the immune system; thus, lymphocytes are able to recognize non-self antigens without the need of any adjuvant, but, in the absence of this, the immune response to vaccination is rather inefficient as if these crude extracts were essential for initiating the body's immune response. This being the case, even the noun "adjuvant", from Latin adiuvare (to help) seems inadequate as it appears that rather than merely "helping" adjuvants are indeed essential. Among the different mechanisms proposed to elucidate the role of adjuvants at the molecular level, we wish to highlight activation and maturation of antigen presenting cells (APC) that migrate to the draining lymph nodes and activation of inflammasomes [27]. These mechanisms bear clinical relevance in the context of cancer vaccines where adjuvants similar in principle to imuno ${ }^{\mathrm{TM}}$ are considered. Thus, it can be hypothesized that the most efficient way to take advantage of activated lymphocytes and cells of the innate arm of the immune system, could be intradermal injection of the cancer vaccine together with its adjuvant in an anatomical location where the flow of lymph would carry the activated immune cells toward the malignancy.

It is worth noticing that intradermal delivery of vaccines is considered one of the best ways to achieve immunization as it has been demonstrated that it generates a higher response with lower dose when associated with a suitable adjuvant [28]. In addition, it has been highlighted that intradermal vaccines containing adjuvants similar in principle to imuno ${ }^{\mathrm{TM}}$ offer the advantage that they could be self-administered and sent through the mail since there is no need for long needles or technical expertise to achieve effective immunization. According to Authors from USA, Israel and Canada in reference to an intradermal flu vaccine containing a new adjuvant based on 1,2-Dipalmitoyl-sn-glycero-3-phosphocholine - a type of phosphatidylcholine - in the event of a pandemic outbreak, intradermal self-administration of vaccines sent by mail "could alleviate the congregation of patients in health centers and thus reduce the potential of these centers to enhance the spread of lethal infection" [28]. In the case of the study quoted above, 1,2-Dipalmitoyl-sn-glycero-3-phosphocholine was conjugated with a Toll-like receptor 4 (TLR4) ligand. In the case of imuno ${ }^{\mathrm{TM}}$, phosphatidylcholine is complexed with low molecular weight microbial chondroitin sulfate and vitamin $D_{3}$ and it is known that vitamin $D_{3}$ regulates human dendritic cell response synergistically with Toll-like receptor agonists [29]. Because of this similarity, it can be hypothesized that imuno ${ }^{\mathrm{TM}}$ may be compounded in intradermal preparations that can be sent through the mail and self-administered in analogy with the strategy proposed by Carter et al. [28]. 


\section{Conclusions}

It is foreseeable that cancer vaccines will become an essential tool in the oncologist's armamentarium and, in analogy with traditional vaccines designed to prevent infectious diseases, effective adjuvants will be required to maximize the effects of vaccination. With the design of imuno $^{\mathrm{TM}}$ for the first time, we have in a single supramolecular structure the features of Freund's-like water and oil emulsions and those of polysaccharide-based adjuvants. In addition, imuno $^{\mathrm{TM}}$ can be considered a delivery system analogous to that proposed for HIV DNA vaccines [25], thus performing several functions, all aimed at optimizing immunization. Considering its role as a delivery system, we propose that imuno $^{\mathrm{TM}}$ may also be considered in the context of mucosal immunization that is a promising novel strategy for fighting pathogens and cancer at mucosal sites [30].

\section{Authors' contribution}

The Authors contributed to the genesis and development of the project described in this study and to the writing of the manuscript.

\section{Ethics}

This article is original and contains unpublished material.

\section{Conflict of interest}

Marco Ruggiero is the founder and CEO of Silver Spring, a Swiss company dedicated to research, development, and production of supplements and probiotics. No product of Silver Spring is mentioned in this article. Marco Ruggiero has invented a number of products including the product designated imuno ${ }^{\mathrm{TM}}$ here described and consults for several companies. Marco Ruggiero is a member of the Editorial Board of The Madridge Journal of Vaccines; he receives no remuneration for his editorial work. Stefania Pacini has invented the product designated imuno ${ }^{\mathrm{TM}}$ here described and consults for several companies.

\section{Advisory}

No information in this paper is presented by the authors as medical advice. Caregivers, researchers, and interested parties should research all information given. Beginning any significant biomedical or other interventions that may impact physiology or making changes to an established regimen should be discussed with the patient's physician in advance. Standard of care for each pathology must be followed as well as rules and regulations established by Health Authorities of each Country.

\section{References}

1. Krone B, Kölmel KF, Grange JM. The biography of the immune system and the control of cancer: from St Peregrine to contemporary vaccination strategies. BMC Cancer. 2014; 14: 595. doi: 0.1186/1471-2407-14-595

2. Kucerova $P$, Cervinkova M. Spontaneous regression of tumour and the role of microbial infection - possibilities for cancer treatment. Anti-Cancer Drugs. 2016; 27(4): 269-277. doi: 10.1097/CAD.0000000000000337
3. Liu JKH. Anti-Cancer Vaccines - A One-Hit Wonder? Yale J Biol Med. 2014 87(4): 481-489.

4. Finn OJ, Khleif SN, Herberman RB. The FDA Guidance on therapeutic cancer vaccines: the need for revision to include preventive cancer vaccines or for a new guidance dedicated to them. Cancer prevention research. 2015; 8(11): 1011-1016. doi: 10.1158/1940-6207.CAPR-15-0234

5. Cheever MA, Higano CS. PROVENGE (Sipuleucel-T) in Prostate Cancer: The First FDA-Approved Therapeutic Cancer Vaccine. Clin Cancer Res. 2011; 17(11): 3520-3526. doi: 10.1158/1078-0432.CCR-10-3126

6. Berti A, Stefani $M$, Degl'Innocenti $D$, et al. Effect of exogenously added acylphosphatases on inositol lipid metabolism in human platelets. FEBS Lett. 1988; 235(1-2): 229-32.

7. Ruggiero $M$, Pazzagli $C$, Rigacci $S$, et al. Negative growth control by a novel low $M(r)$ phosphotyrosine protein phosphatase in normal and transformed cells. FEBS Lett. 1993; 326(1-3): 294-8.

8. Pierce JH, Di Marco E, Cox GW, Lombardi D, et al. Macrophage-colonystimulating factor (CSF-1) induces proliferation, chemotaxis, and reversible monocytic differentiation in myeloid progenitor cells transfected with the human c-fms/CSF-1 receptor cDNA. Proc Natl Acad Sci USA. 1990; 87(15): 5613-7.

9. Ruggiero $M$, Ward E, Smith $R$, et al. Oleic Acid, deglycosylated vitamin D-binding protein, nitric oxide: a molecular triad made lethal to cancer. Anticancer Res. 2014; 34(7): 3569-78.

10. Freund J, McDermott K. Sensitization to Horse Serum by Means of Adjuvants. Exp Biol Med. 1942; 49(4): 548-553. doi: 10.1016/S00218707(42)90209-0

11. Banday AH, Jeelani S, Hruby VJ. Cancer vaccine adjuvants - recent clinical progress and future perspectives. Immunopharmacol Immunotoxicol. 2015; 37(1): 1-11. doi: 10.3109/08923973.2014.971963

12. Lai RP, Seaman MS, Tonks $P$, et al. Mixed adjuvant formulations reveal a new combination that elicit antibody response comparable to Freund's adjuvants. PLoS One. 2012; 7(4): e35083. doi: 10.1371/journal.pone.0035083

13. Li X, Min M, Du N, et al. Chitin, Chitosan, and Glycated Chitosan Regulate Immune Responses: The Novel Adjuvants for Cancer Vaccine. Clin Dev Immunol. 2013; 2013: 387023. doi: 10.1155/2013/387023

14. Prudden JF. The treatment of human cancer with agents prepared from bovine cartilage. J Biol Response Mod. 1985; 4(6): 551-84.

15. Saburi E, Saburi A, Ghanei M. Promising role for Gc-MAF in cancer immunotherapy: from bench to bedside. Caspian J Intern Med. 2017; 8(4): 228-238. doi: 10.22088/cjim.8.4.228

16. Yamamoto N. Structural definition of a potent macrophage activating factor derived from vitamin D3-binding protein with adjuvant activity for antibody production. Mol Immunol. 1996; 33(15): 1157-64.

17. Thyer L, Ward E, Smith R, et al. A Novel Role for a Major Component of the Vitamin D Axis: Vitamin D Binding Protein-Derived Macrophage Activating Factor Induces Human Breast Cancer Cell Apoptosis through Stimulation of Macrophages. Nutrients. 2013; 5(7): 2577-2589. doi: 10.3390/nu5072577

18. Ruggiero $M$, Reinwald $H$, Pacini $S$. Is chondroitin sulfate responsible for the biological effects attributed to the GC protein-derived Macrophage Activating Factor (GcMAF)? Med Hypotheses. 2016; 94: 126-31. doi: 10.1016/j.mehy.2016.07.012

19. Peter T, Ruggiero M. Ketogenic Diet and Immunotherapy in Cancer and Neurological Diseases. 4th International Congress on Integrative Medicine, 1, 2 April 2017, Fulda, Germany. Am J Immunol. 2017; 13: 158164. doi: 10.3844/ajisp.2017.158.164

20. Schwalb M, Taubmann M, Hines S, et al. Clinical Observation of a Novel, Complementary, Immunotherapeutic Approach based on Ketogenic Diet, Chondroitin Sulfate, Vitamin D3, Oleic Acid and a Fermented Milk and Colostrum Product. Am J Immunol. 2016; 12: 91-98. doi: 10.3844/ ajisp.2016.91.98

21. Bauerova $K$, Ponist $S$, Kuncirova $V$, et al. Effect of nonanimal high- and low-molecular-mass chondroitin sulfates produced by a biotechnological process in an animal model of polyarthritis. Pharmacology. 2014; 94(3-4): 109-14. doi: 0.1159/000366285 
22. Robert F. The potential benefits of low-molecular-weight heparins in cancer patients. J Hematol Oncol. 2010; 3: 3. doi: 10.1186/1756-8722-3-3

23. Vannucchi S, Ruggiero M, Chiarugi V. Complexing of heparin with phosphatidylcholine. A possible supramolecular assembly of plasma heparin. Biochem J. 1985; 227(1): 57-65

24. Yanagawa $\mathrm{H}$, Ogawa $\mathrm{Y}, \mathrm{Kojima} \mathrm{K}$, et al. Construction of protocellular structures under simulated primitive earth conditions. Orig Life Evol Biosph. 1988; 18(3): 179-207.

25. Ruggiero M. A Novel Method to Enhance Immune Responses Induced by HIV DNA Vaccination. BAOJ HIV. 2017; 3: 027

26. Gayed PM. Toward a Modern Synthesis of Immunity: Charles A. Janeway Jr. and the Immunologist's Dirty Little Secret. Yale J Biol Med. 2011; 84(2): 131-138
27. Awate S, Babiuk LA, Mutwiri G. Mechanisms of Action of Adjuvants Frontiers in Immunology. 2013; 4: 114. doi: 10.3389/fimmu.2013.00114

28. Carter $D$, van Hoeven $N$, Baldwin $\mathrm{S}$, et al. The adjuvant GLA-AF enhances human intradermal vaccine responses. Sci Adv. 2018; 4: eaas9930. doi: $10.1126 /$ sciadv.aas9930

29. Brosbøl-Ravnborg A, Bundgaard B, Höllsberg P. Synergy between Vitamin D3 and Toll-Like Receptor Agonists Regulates Human Dendritic Cell Response during Maturation. Clin Dev Immunol. 2013; 807971: 8. doi: $10.1155 / 2013 / 807971$

30. Nizard M, Diniz MO, Roussel H, et al. Mucosal vaccines: Novel strategies and applications for the control of pathogens and tumors at mucosal sites. Hum Vaccin Immunother. 2014; 10(8): 2175-2187. doi: 10.4161/ hv.29269 